\title{
On the direct sum of two bounded linear operators and subspace-hypercyclicity
}

\author{
Nareen Bamernia,*, Adem Kılıçman ${ }^{b}$ \\ a Department of Mathematics, University of Duhok, Kurdistan Region, Iraq. \\ ${ }^{b}$ Department of Mathematics, University Putra Malaysia, 43400 UPM, Serdang, Selangor, Malaysia.
}

\begin{abstract}
In this paper, we study the relation between subspace-hypercyclicity and the direct sum of two operators. In particular, we show that if the direct sum of two operators is subspace-hypercyclic, then both operators are subspace-hypercyclic; however, the converse is true for a stronger property than subspace-hypercyclicity. Moreover, we prove that if an operator T satisfies subspace-hypercyclic criterion, then $T \oplus T$ is subspace-hypercyclic. However, we show that the converse is true under certain conditions.@2019 All rights reserved.
\end{abstract}

Keywords: Hypercyclic operators, Direct sums. 2010 MSC: MSC 47A16.

\section{Introduction}

A bounded linear operator $T$ on a separable Banach space $X$ is hypercyclic if there is a vector $x \in X$ such that $\operatorname{Orb}(T, x)=\left\{T^{n} x: n \geqslant 0\right\}$ is dense in $X$, such a vector $x$ is called hypercyclic for $T$. The first example of a hypercyclic operator on a Banach space was constructed by Rolewicz in 1969 [11]. He showed that, if $B$ is the backward shift on $\ell^{\mathfrak{p}}(\mathbb{N})$ then $\lambda B$ is hypercyclic if and only if $|\lambda|>1$.

In 2011, Madore and Martínez-Avendaño [9] considered the density of the orbit in a non-trivial subspace instead of the whole space, such a concept is called subspace-hypercyclicity. An operator is subspacehypercyclic for a subspace $\mathcal{M}$ of $\mathcal{X}$ (or $\mathcal{X}$-hypercyclic, for short) if there exists a vector $x \in \mathcal{X}$ whose orbit is dense in $\mathcal{M}$. Also, Madore and Martínez-Avendaño [9] defined subspace-transitive operators and showed that every subspace-transitive is subspace-hypercyclic. Talebi and Moosapoor [13] defined subspace-mixing concept which is a stronger property than subspace-transitive. For more information on subspace-hypercyclicity, the reader may refer to $[8,10,1]$.

In 1982, Kitai [7] showed that if $T_{1} \oplus T_{2}$ is hypercyclic, then $T_{1}$ and $T_{2}$ are hypercyclic. For subspacehypercyclicity, Madore and Martínez-Avendaño showed that there exists an operator $T$ on a Banach space $X$ such that $\mathrm{T} \oplus \mathrm{I}$ is subspace-hypercyclic operator for the subspace $X \oplus\{0\}$ [9, Example 2.2]. However, it is

\footnotetext{
*Corresponding author

Email addresses: nareen_bamerni@yahoo.com (Nareen Bamerni), akilicman@yahoo.com (Adem K1lıçman)
} 
clear that the identity operator I cannot be subspace-hypercyclic for any nontrivial subspace. Therefore, Kitai's result cannot be extended to subspaces. On the other hand, for nontrivial subspaces and operators, we have the following question:

Question 1.1. If the direct sum of two operators is subspace-hypercyclic, are both operators subspace-hypercyclic?

De la Rosa and Read [6] showed that the converse of Kitai's result is not true by giving a hypercyclic operator $\mathrm{T}$ such that $\mathrm{T} \oplus \mathrm{T}$ is not. This yields to ask the analogous question for subspace-hypercyclic operators. In particular, we have

Question 1.2. If two operators are subspace-hypercyclic, is their direct sum subspace-hypercyclic?

On the other hand, if T satisfies hypercyclic criterion, then $T \oplus T$ is hypercyclic [3]. In 1999, Bés and Peris [4] proved that the converse is also true; i.e, if $\mathrm{T} \oplus \mathrm{T}$ is hypercyclic, then $\mathrm{T}$ satisfies hypercyclic criterion. Now, it is natural to ask analogous questions for subspace-hypercyclic operators. In particular, we have the following questions:

Question 1.3. If T satisfies subspace-hypercyclic criterion, is $\mathrm{T} \oplus \mathrm{T}$ subspace-hypercyclic?

Question 1.4. If $\mathrm{T} \oplus \mathrm{T}$ is subspace-hypercyclic, does $\mathrm{T}$ satisfy hypercyclic criterion?

In the second section of this paper, we answer the Question 1.1 positively; i.e, if $T_{1} \oplus T_{2}$ is subspacehypercyclic, then $T_{1}$ and $T_{2}$ are subspace-hypercyclic. Moreover, we show that Question 1.2 has a partial positive answer. In particular, if two operators are subspace-transitive and at least one of them is subspacemixing, then their direct sum is subspace-transitive which in turn is subspace-hypercyclic. Also, we show that if the direct sum of two operators satisfies subspace-hypercyclic criterion, then both operators do. However, we give counterexamples to show that the converse is not true in general. On the other hand, we show the converse is only true for a single operator; i.e, if T satisfies subspace-hypercyclic criterion, then $T \oplus T$ does, which gives a positive answer to the Question1.3. Furthermore, we show that under certain conditions if $T \oplus T$ is subspace-hypercyclic, then $T$ satisfies subspace-hypercyclic criterion which answers Question 1.4 partially.

Let $\mathcal{M}_{1}$ and $\mathcal{M}_{2}$ be subspaces of a Banach space $\mathcal{X}$, then the direct sum of $\mathcal{M}_{1}$ and $\mathcal{M}_{2}$ is defined as follows

$$
\mathcal{M}_{1} \oplus \mathcal{M}_{2}=\left\{(x, y): x \in \mathcal{M}_{1}, y \in \mathcal{M}_{2}\right\}
$$

For more information and details on the direct sum of Banach spaces, the reader may refer to [5]. Let $\left\{e_{i}: e_{i}=(\cdots, 0,1,0, \cdots), i \in \mathbb{Z}\right\}$ be a standard basis for the sequence space $\ell^{2}(\mathbb{Z})$, then we have the following theorems.

Theorem 1.5. [2] Let $\mathrm{T}$ be an invertible bilateral forward weighted shift in $\ell^{2}(\mathbb{Z})$ with a positive weight sequence $\left\{w_{n}\right\}_{\mathfrak{n} \in \mathbb{Z}}$ and $\mathcal{M}$ be a subspace of $\ell^{2}(\mathbb{Z})$. Then $\mathrm{T}$ is $\mathcal{\mathcal { M }}$-transitive if and only if there exist an increasing sequence of positive integers $\left\{n_{k}\right\}_{k \in \mathcal{N}}$ and $e_{m_{i}} \in\left\{e_{r}\right\} \cap \mathcal{M}$ such that $T^{n_{k}} \mathcal{M} \subseteq \mathcal{M}$ for all $k \in \mathcal{N}$ and

$$
\lim _{k \rightarrow \infty} \prod_{j=m_{i}}^{m_{i}+n_{k}-1} w_{j}=0 \text { and } \lim _{k \rightarrow \infty} \prod_{j=1+m_{i}}^{n_{k}+m_{i}} \frac{1}{w_{-j}}=0
$$

Theorem 1.6. [2] Let $T_{1}$ and $T_{2}$ be invertible bilateral forward weighted shifts in $\ell^{2}(\mathbb{Z})$ with a positive weight sequence $\left\{w_{n}\right\}_{n \in \mathbb{Z}}$ and $\left\{a_{n}\right\}_{n \in \mathbb{Z}}$, respectively. Let $\mathcal{M}_{1}$ and $\mathcal{M}_{2}$ be closed subspaces of $\ell^{2}(\mathbb{Z})$. Then $T_{1} \oplus T_{2}$ is $\mathcal{M}_{1} \oplus \mathcal{M}_{2}$-transitive if and only if there exist $e_{m_{i}} \in\left\{e_{r}\right\} \cap \mathcal{M}_{1}, e_{h_{p}} \in\left\{e_{r}\right\} \cap \mathcal{M}_{2}$ and an increasing sequence of positive integers $\left\{n_{k}\right\}_{k \in \mathcal{N}}$ such that $\left(\mathrm{T}_{1} \oplus \mathrm{T}_{2}\right)^{\mathrm{n}_{\mathrm{k}}}\left(\mathcal{M}_{1} \oplus \mathcal{M}_{2}\right) \subseteq \mathcal{M}_{1} \oplus \mathcal{M}_{2}$ for all $\mathrm{k} \in \mathcal{N}$ and

$$
\lim _{k \rightarrow \infty} \max \left\{\prod_{j=m_{i}}^{m_{i}+n_{k}-1} w_{j}, \prod_{j=h_{p}}^{h_{p}+n_{k}-1} a_{j}\right\}=0
$$


and

$$
\lim _{k \rightarrow \infty} \max \left\{\prod_{j=1-m_{i}}^{n_{k}-m_{i}} \frac{1}{w_{-j}}, \prod_{j=1-h_{p}}^{n_{k}-h_{p}} \frac{1}{a_{-j}}\right\}=0
$$

\section{Main Result}

In this paper, all Banach spaces are infinite dimensional separable over the field $\mathbb{C}$ of complex numbers. We always assume that a subspace $\mathcal{M}$ of a Banach space $\mathcal{X}$ is nontrivial $(\mathcal{M} \neq \mathcal{X}$ and $\mathcal{M} \neq\{0\})$ and topologically closed. Also, we assume that every bounded linear operator $\mathrm{T}$ is nontrivial $(\mathrm{T} \neq \mathrm{I}$ and $\mathrm{T} \neq 0$, where $I$ is identity operator and 0 is zero operator) unless otherwise stated. We denote $H C(T, \mathcal{M})$ the set of all $\mathcal{M}$-hypercyclic vectors for $\mathrm{T}$.

Proposition 2.1. Let $\mathrm{T} \in \mathcal{B}(\mathcal{H})$, and $\mathcal{M}$ be a closed subspace of $\mathcal{H}$. The following statements are equivalent:

1. $\mathrm{T}$ is $\mathcal{M}$-transitive,

2. for each $x, y \in \mathcal{M}$, there exist sequences $\left\{x_{k}\right\}_{k \in \mathcal{N}} \subset \mathcal{M}$ and an increasing sequence of positive integers $\left\{n_{k}\right\}_{k \in \mathcal{N}}$ such that $\mathrm{T}^{n_{k}} \mathcal{M} \subseteq \mathcal{M}$ for all $\mathrm{k} \geqslant 1, \mathrm{x}_{\mathrm{k}} \rightarrow \mathrm{x}$ and $\mathrm{T}^{\mathrm{n}_{\mathrm{k}}} \mathrm{x}_{\mathrm{k}} \rightarrow \mathrm{y}$ as $\mathrm{k} \rightarrow \infty$,

3. for each $x, y \in \mathcal{M}$ and each 0 -neighborhood $W$ in $\mathcal{M}$, there exist $z \in \mathcal{M}$ and $\mathrm{n} \in \mathcal{N}$ such that $x-z \in W$, $\mathrm{T}^{\mathrm{n}} z-\mathrm{y} \in \mathrm{W}$ and $\mathrm{T}^{\mathrm{n}} \mathcal{M} \subseteq \mathcal{M}$.

Proof. (1) $\Rightarrow(2)$ : Let $x, y \in \mathcal{M}$. For all $k \geqslant 1$, suppose that $B_{k}=\mathbb{B}(x, 1 / k)$ and $B_{k}^{\prime}=\mathbb{B}(y, 1 / k)$ are two open balls in $\mathcal{X}$, then $A_{k}=B_{k} \cap \mathcal{M}$ and $A_{k}^{\prime}=B_{k}^{\prime} \cap \mathcal{M}$ are relatively open subsets of $\mathcal{M}$. By [9, Theorem 3.3.], there exist sequence $\left\{n_{k}\right\}$ in $\mathbb{N}$ and $\left\{x_{k}\right\}$ in $\mathcal{M}$ such that for all $k \geqslant 1$,

$$
x_{k} \in T^{-n_{k}}\left(A_{k}^{\prime}\right) \cap A_{k} \text { and } T^{n_{k}} \mathcal{M} \subseteq \mathcal{M} \text {. }
$$

It follows that

$$
x_{k} \in A_{k} \text { and } T^{n_{k}}\left(x_{k}\right) \in A_{k}^{\prime} .
$$

Then, as $k \rightarrow \infty$ the desired result follows.

$(2) \Rightarrow(3)$ : Follows immediately from part (2) by taking $z=x_{k}$ and $n_{k}=n$ for a large enough $k \in \mathbb{N}$.

$(3) \Rightarrow(1)$ : Let $U$ and $V$ be two nonempty open subset of $\mathcal{M}$. Let $W$ be a neighborhood for zero, pick $x \in U$ and $y \in V$, so there exist $z \in \mathcal{M}$ and $n \in \mathbb{N}$ such that $x-z \in W, T^{n}(z)-y \in W$ and $T^{n} \mathcal{M} \subseteq \mathcal{M}$. It follows that $\mathrm{U} \cap \mathrm{T}^{-\mathrm{n}}(\mathrm{V}) \neq \phi$ which proves (1), by [9, Theorem 3.3.].

The following theorem gives a positive answer to Question 1.1.

Theorem 2.2. Let $\mathcal{M}_{1}$ and $\mathcal{M}_{2}$ be closed subspaces of $\mathcal{X}$ and $\mathrm{T}_{1} \oplus \mathrm{T}_{2}$ is $\left(\mathcal{M}_{1} \oplus \mathcal{M}_{2}\right)$-hypercyclic, then $\mathrm{T}_{1}$ and $\mathrm{T}_{2}$ are $\mathcal{M}_{1}$-hypercyclic and $\mathcal{M}_{2}$-hypercyclic, respectively.

Proof. Let $a \in \mathcal{M}_{1}$ and $b \in \mathcal{M}_{2}$, and let $(x, y) \in \mathrm{HC}\left(\mathrm{T}_{1} \oplus \mathrm{T}_{2}, \mathcal{M}_{1} \oplus \mathcal{M}_{2}\right)$, then there exist an $\epsilon>0$ and an increasing sequence of positive integers $\left\{n_{k}\right\}_{k \in \mathbb{N}}$ such that

$$
\left\|\left(T_{1} \oplus T_{2}\right)^{n_{k}}(x, y)-(a, b)\right\|_{\mathcal{M}_{1} \oplus \mathcal{M}_{2}}^{2} \leqslant \epsilon .
$$

It follows that,

$$
\left\|T_{1}^{n_{k}} x-a\right\|_{\mathcal{M}_{1}}^{2}+\left\|T_{2}^{n_{k}} y-b\right\|_{\mathcal{M}_{2}}^{2} \leqslant \epsilon
$$

Then

$$
\left\|T_{1}^{n_{k}} x-a\right\|_{\mathcal{M}_{1}} \leqslant \epsilon \text { and }\left\|T_{2}^{n_{k}} y-b\right\|_{\mathcal{M}_{2}} \leqslant \epsilon .
$$

Thus, there exists an increasing sequence of positive integers $\left\{n_{k}\right\}_{k \in \mathbb{N}}$ such that $\left\{T_{1}^{n_{k}} x: k \geqslant 1\right\}$ and $\left\{T_{2}^{\mathfrak{n}_{k}} y: k \geqslant 1\right\}$ are dense in $\mathcal{M}_{1}$ and $\mathcal{M}_{2}$, respectively. Therefore $\operatorname{Orb}\left(T_{1}, x\right)$ and $\operatorname{Orb}\left(T_{2}, y\right)$ are dense in $\mathcal{M}_{1}$ and $\mathcal{M}_{2}$, respectively. 
The following two results show that the converse of Theorem 2.2 holds true under some conditions, which gives a partial positive answer to Question 1.2.

Theorem 2.3. If $\mathrm{T}_{1}$ and $\mathrm{T}_{2}$ are $\mathcal{M}_{1}$-transitive and $\mathcal{M}_{2}$-transitive, respectively, and at least one of them is subspacemixing, then $\mathrm{T}_{1} \oplus \mathrm{T}_{2}$ is $\left(\mathcal{M}_{1} \oplus \mathcal{M}_{2}\right)$-transitive.

Proof. Suppose that, without loss of generality, that $T_{1}$ is $\mathcal{M}_{1}$-mixing. Let $U_{1} \oplus V_{1}$ and $U_{2} \oplus V_{2}$ be open sets in $\mathcal{M}_{1} \oplus \mathcal{M}_{2}$, then $U_{1}$ and $U_{2}$ are open in $\mathcal{M}_{1}$, and $V_{1}$ and $V_{2}$ are open in $\mathcal{M}_{2}$. By hypothesis, there exist two numbers $\mathrm{N}_{1}, \mathrm{~N}_{2} \in \mathbb{N}$ such that

$$
\mathrm{T}_{1}^{-\mathrm{n}}\left(\mathrm{U}_{1}\right) \cap \mathrm{U}_{2} \neq \phi \text { and } \mathrm{T}_{1}^{\mathrm{n}}\left(\mathcal{M}_{1}\right) \subseteq \mathcal{M}_{1} \text { for all } \mathrm{n} \geqslant \mathrm{N}_{1}
$$

and

$$
\mathrm{T}_{2}^{-\mathrm{N}_{2}}\left(\mathrm{~V}_{1}\right) \cap \mathrm{V}_{2} \neq \phi \text { and } \mathrm{T}_{2}^{\mathrm{N}_{2}}\left(\mathcal{M}_{2}\right) \subseteq \mathcal{M}_{2}
$$

Since $T_{2}$ is $\mathcal{M}_{2}$-transitive, then $\left\{n \in \mathbb{N}: T_{2}^{-n} V_{1} \cap V_{2}\right.$ and $\left.T_{2}^{n} \mathcal{M}_{2} \subseteq \mathcal{M}_{2}\right\}$ is infinite [12]. So, there exists $p \in \mathbb{N}$ such that

$$
\mathrm{T}_{1}^{-\mathrm{p}}\left(\mathrm{U}_{1}\right) \cap \mathrm{U}_{2} \neq \phi, \mathrm{T}_{2}^{-\mathrm{p}}\left(\mathrm{V}_{1}\right) \cap \mathrm{V}_{2} \neq \phi, \mathrm{T}_{1}^{\mathrm{p}}\left(\mathcal{M}_{1}\right) \subseteq \mathcal{M}_{1} \text { and } \mathrm{T}_{2}^{\mathrm{p}}\left(\mathcal{M}_{2}\right) \subseteq \mathcal{M}_{2} \text {. }
$$

It follows that

$$
\left(\mathrm{T}_{1} \oplus \mathrm{T}_{2}\right)^{-\mathrm{p}}\left(\mathrm{U}_{1} \oplus \mathrm{V}_{1}\right) \cap\left(\mathrm{U}_{2} \oplus \mathrm{V}_{2}\right) \neq \phi \text { and }\left(\mathrm{T}_{1} \oplus \mathrm{T}_{2}\right)^{\mathrm{p}}\left(\mathcal{M}_{1} \oplus \mathcal{M}_{2}\right) \subseteq\left(\mathcal{M}_{1} \oplus \mathcal{M}_{2}\right)
$$

Thus, $\mathrm{T}_{1} \oplus \mathrm{T}_{2}$ is $\left(\mathcal{M}_{1} \oplus \mathcal{M}_{2}\right)$-transitive.

Proposition 2.4. Let $\mathcal{M}_{1}$ and $\mathcal{M}_{2}$ be closed subspaces of $\mathcal{X}$, then $\mathrm{T}_{1}$ and $\mathrm{T}_{2}$ are $\mathcal{M}_{1}$-mixing and $\mathcal{M}_{2}$-mixing; respectively, if and only if $\mathrm{T}_{1} \oplus \mathrm{T}_{2}$ is $\left(\mathcal{M}_{1} \oplus \mathcal{M}_{2}\right)$-mixing.

Proof. For the "if" part, let $\mathrm{U}_{1}$ and $\mathrm{U}_{2}$ be open sets in $\mathcal{M}_{1}$, and $\mathrm{V}_{1}$ and $\mathrm{V}_{2}$ be open sets in $\mathcal{M}_{2}$, then $\mathrm{U}_{1} \oplus \mathrm{V}_{1}$ and $\mathrm{U}_{2} \oplus \mathrm{V}_{2}$ are open in $\mathcal{M}_{1} \oplus \mathcal{M}_{2}$. So there exists an $\mathrm{N} \in \mathbb{N}$ such that

$$
\left(\mathrm{T}_{1} \oplus \mathrm{T}_{2}\right)^{-\mathrm{n}}\left(\mathrm{U}_{1} \oplus \mathrm{V}_{1}\right) \cap\left(\mathrm{U}_{2} \oplus \mathrm{V}_{2}\right) \neq \phi
$$

and

$$
\left(\mathrm{T}_{1} \oplus \mathrm{T}_{2}\right)^{\mathrm{n}}\left(\mathcal{M}_{1} \oplus \mathcal{M}_{2}\right) \subseteq\left(\mathcal{M}_{1} \oplus \mathcal{M}_{2}\right)
$$

for all $n \geqslant N$. Then

$$
\mathrm{T}^{-\mathrm{n}}\left(\mathrm{U}_{1}\right) \cap \mathrm{U}_{2} \neq \phi, \mathrm{T}^{-\mathrm{n}}\left(\mathrm{V}_{1}\right) \cap \mathrm{V}_{2} \neq \phi, \mathrm{T}^{\mathrm{n}}\left(\mathcal{M}_{1}\right) \subseteq \mathcal{M}_{1} \text { and } \mathrm{T}^{\mathrm{n}}\left(\mathcal{M}_{2}\right) \subseteq \mathcal{M}_{2}
$$

Therefore, $T_{1}$ and $T_{2}$ are $\mathcal{M}_{1}$-mixing and $\mathcal{M}_{2}$-mixing, respectively.

The proof of the "only if" part is similar to the proof of Theorem 2.3.

Proposition 2.5. If $\mathrm{T}_{1} \oplus \mathrm{T}_{2}$ satisfies $\left(\mathcal{M}_{1} \oplus \mathcal{M}_{2}\right)$-hypercyclic criterion, then $\mathrm{T}_{1}$ and $\mathrm{T}_{2}$ satisfy $\mathcal{M}_{1}$-hypercyclic criterion and $\mathcal{M}_{2}$-hypercyclic criterion, respectively.

Proof. By hypothesis, there exist two dense sets of the form $\mathrm{D}_{1} \oplus \mathrm{D}_{2}$ and $\mathrm{D}_{3} \oplus \mathrm{D}_{4}$ in $\mathcal{M}_{1} \oplus \mathcal{M}_{2}$ and an increasing sequence of positive integers $\left\{n_{k}\right\}_{k \in \mathbb{N}}$ such that

(i) $\left(T_{1} \oplus T_{2}\right)^{n_{k}}\left(x_{1}^{\prime}, x_{2}^{\prime}\right) \rightarrow(0,0)$ for all $\left(x_{1}^{\prime}, x_{2}^{\prime}\right) \in D_{1} \oplus D_{2}$.

(ii) for each $\left(y_{1}^{\prime}, y_{2}^{\prime}\right) \in D_{3} \oplus D_{4}$ there exists a sequence $\left\{\left(x_{k}, y_{k}\right)\right\}_{k \in \mathbb{N}} \subset \mathcal{M}_{1} \oplus \mathcal{M}_{2}$ such that $\left(x_{k}, y_{k}\right) \rightarrow 0$ and $\left(T_{1} \oplus T_{2}\right)^{n_{k}}\left(x_{k}, y_{k}\right) \rightarrow\left(y_{1}^{\prime}, y_{2}^{\prime}\right)$. 
Since $D_{1}$ and $D_{3}$ are dense sets in $\mathcal{M}_{1}$, and $D_{2}$ and $D_{4}$ are dense sets in $\mathcal{M}_{2}$, then it is easy to show that

(i) $T_{1}^{n_{k}} x_{1}^{\prime} \rightarrow 0$ for all $x_{1}^{\prime} \in D_{1}$ and $T_{2}^{n_{k}} x_{2}^{\prime} \rightarrow 0$ for all $x_{2}^{\prime} \in D_{2}$,

(ii) for each $y_{1}^{\prime} \in D_{3}$ there exists a sequence $\left\{x_{k}\right\}_{k \in \mathbb{N}} \subset \mathcal{M}_{1}$ such that $x_{k} \rightarrow 0$ and $T_{1}^{n_{k}} x_{k} \rightarrow y_{1}^{\prime}$, and for each $y_{2}^{\prime} \in D_{4}$ there exists a sequence $\left\{y_{k}\right\}_{k \in \mathbb{N}} \subset \mathcal{M}_{2}$ such that $y_{k} \rightarrow 0$ and $T_{2}^{n_{k}} y_{k} \rightarrow y_{2}^{\prime}$.

it is readily seen that $T_{1}$ and $T_{2}$ satisfy $\mathcal{M}_{1}$-hypercyclic criterion and $\mathcal{M}_{2}$-hypercyclic criterion, respectively.

The following two examples show that the converse of Proposition 2.5 is not true in general.

Example 2.6. Let $\mathcal{M}_{1}$ and $\mathcal{M}_{2}$ be two subspaces of $X$ such that $\mathcal{M}_{1} \oplus \mathcal{M}_{2} \neq X$. If for any two increasing sequences of positive integers $\left\{n_{k}\right\}_{k \in \mathbb{N}}$ and $\left\{m_{k}\right\}_{k \in \mathbb{N}}$, we have $\left\{n_{k}\right\}_{k \in \mathbb{N}} \cap\left\{m_{k}\right\}_{k \in \mathbb{N}}=\phi$ whenever $T_{1}$ satisfies $\mathcal{M}_{1}$-hypercyclic criterion with respect to $\left\{\mathrm{n}_{\mathrm{k}}\right\}_{\mathrm{k} \in \mathbb{N}}$ and $\mathrm{T}_{2}$ satisfies $\mathcal{M}_{2}$-hypercyclic criterion with respect to $\left\{\mathrm{m}_{\mathrm{k}}\right\}_{\mathrm{k} \in \mathbb{N}}$. Then, $\mathrm{T}_{1} \oplus \mathrm{T}_{2}$ does not satisfy $\left(\mathcal{M}_{1} \oplus \mathcal{M}_{2}\right)$-hypercyclic criterion.

Example 2.7. Let $\mathcal{M}_{1}$ and $\mathcal{M}_{2}$ be closed subspaces of the Banach space $\ell^{\mathfrak{p}}(\mathbb{Z})$. Choose sequences $\left\{w_{n}\right\}_{n \in \mathbb{Z}}$ and $\left\{a_{n}\right\}_{\mathfrak{n} \in \mathbb{Z}}$ of positive real numbers such that each one satisfies Theorem 1.5 but not either ((1.2)) nor ((1.3)) of Theorem 1.6. Then, suppose that $T_{1}$ and $T_{2}$ are bilateral weighted shifts with weight sequence $\left\{w_{n}\right\}_{\mathfrak{n} \in \mathbb{Z}}$ and $\left\{a_{n}\right\}_{\mathfrak{n} \in \mathbb{Z}^{\prime}}$ respectively. It follows from Theorem 1.5 and Theorem 1.6 that $\mathrm{T}_{1}$ and $\mathrm{T}_{2}$ satisfy $\mathcal{M}_{1}$-hypercyclic criterion and $\mathcal{M}_{2}$-hypercyclic criterion, respectively. However, $\mathrm{T}_{1} \oplus \mathrm{T}_{2}$ does not satisfy $\left(\mathcal{M}_{1} \oplus \mathcal{M}_{2}\right)$-hypercyclic criterion.

The following proposition shows that the converse of Proposition 2.5 holds true for a single operator $\mathrm{T}$ which gives a positive answer to Question 1.3.

Proposition 2.8. If $\mathrm{T}$ satisfies $\mathcal{M}$-hypercyclic criterion, then $\mathrm{T} \oplus \mathrm{T}$ satisfies $(\mathcal{M} \oplus \mathcal{M})$-hypercyclic criterion.

Proof. Since T satisfies $\mathcal{M}$-hypercyclic criterion, then there exist two dense subsets $\mathrm{D}_{1}$ and $\mathrm{D}_{2}$ of $\mathcal{M}$ and an increasing sequence of positive integers $\left\{n_{k}\right\}_{k \in \mathbb{N}}$ such that all hypothesis of subspace-hypercyclic criterion are satisfied. It is easy to show that $\mathrm{D}_{1} \oplus \mathrm{D}_{1}$ and $\mathrm{D}_{2} \oplus \mathrm{D}_{2}$ are dense sets in $\mathcal{M} \oplus \mathcal{M}$. Let $\left(x_{1}, x_{2}\right) \in \mathrm{D}_{1} \oplus \mathrm{D}_{1}$ then $x_{1}, x_{2} \in D_{1}$. By hypothesis, $T^{n_{k}} x_{1} \rightarrow 0$ and $T^{n_{k}} x_{2} \rightarrow 0$. Thus,

$$
(T \oplus T)^{n_{k}}\left(x_{1}, x_{2}\right) \rightarrow(0,0) .
$$

Now, let $\left(y_{1}, y_{2}\right) \in D_{2} \oplus D_{2}$ then $y_{1}, y_{2} \in D_{2}$. Also, by hypothesis, there exist two sequences say $\left\{x_{k}\right\}_{k \in \mathbb{N}}$ and $\left\{y_{k}\right\}_{k \in \mathbb{N}}$ in $\mathcal{M}$ such that

$$
x_{k} \rightarrow 0 \text { and } T^{n_{k}} x_{k} \rightarrow y_{1}
$$

and

$$
y_{k} \rightarrow 0 \text { and } T^{n_{k}} y_{k} \rightarrow y_{2}
$$

Therefore,

$$
\left(x_{k}, y_{k}\right) \rightarrow(0,0) \text { and }(T \oplus T)^{n_{k}}\left(x_{k}, y_{k}\right) \rightarrow\left(y_{1}, y_{2}\right) .
$$

Finally, since $T^{n_{k}} \mathcal{M} \subseteq \mathcal{M}$, then

$$
(\mathrm{T} \oplus \mathrm{T})^{\mathrm{n}_{\mathrm{k}}}(\mathcal{M} \oplus \mathcal{M}) \subseteq \mathcal{M} \oplus \mathcal{M}
$$

It follows by (2.1), (2.2) and (2.3) that $\mathrm{T} \oplus \mathrm{T}$ satisfies $\mathcal{M} \oplus \mathcal{M}$-hypercyclic criterion, and thus hypercyclic.

Corollary 2.9. If $\mathrm{T}$ satisfies subspace-hypercyclic criterion, then $\mathrm{T} \oplus \mathrm{T}$ is subspace-hypercyclic.

For hypercyclicity, Bés and Peris [4] proved that the converse of the Corollary2.9 above is also true. The next theorem shows that the converse of Corollary 2.9 is true under certain conditions, which gives a partial positive answer to Question 1.4. First we need the following lemma.

Lemma 2.10. Let $\mathrm{T}, \mathrm{S} \in \mathcal{B}(X)$ satisfy the equation $\mathrm{TS}=\mathrm{ST}$ and $\mathcal{M}$ be a subspace of $X$. If $x \in \mathrm{HC}(\mathrm{T}, \mathcal{M})$, then $\mathrm{Sx} \in \mathrm{HC}(\mathrm{T}, \mathrm{SM})$. 
Proof. Let $x \in \operatorname{HC}(T, \mathcal{M})$, then $\operatorname{Orb}(T, x) \cap \mathcal{M}$ is dense in $\mathcal{M}$. Thus

$$
\begin{aligned}
\overline{\operatorname{Orb}(T, S x) \cap S \mathcal{M}} & =\overline{\left\{T^{n} S x: n \geqslant 0\right\} \cap S \mathcal{M}} \\
& =\overline{\left\{T^{n} x: n \geqslant 0\right\} \cap S \mathcal{M}} \\
& =\overline{S\left\{T^{n} x: n \geqslant 0\right\} \cap S \mathcal{M}} \\
& \supseteq \overline{S\left(\left\{T^{n} x: n \geqslant 0\right\} \cap \mathcal{M}\right)} \\
& \supseteq S\left(\overline{\left.\left\{T^{n} x: n \geqslant 0\right\} \cap \mathcal{M}\right)}\right. \\
& =S \mathcal{M} .
\end{aligned}
$$

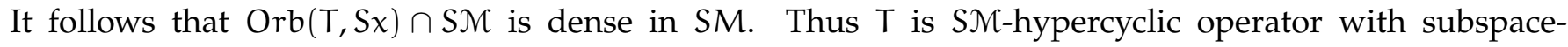
hypercyclic vector $S x$.

Theorem 2.11. Let $\mathrm{T} \oplus \mathrm{T}$ be $(\mathcal{M} \oplus \mathcal{M})$-hypercyclic. If there exists a subspace-hypercyclic vector $(\mathrm{x}, \mathrm{y})$ such that $\mathrm{x}$ has the following properties

(i) $x \in \bigcap_{n \in \mathbb{N}} T^{n}(\mathcal{M})$,

(ii) $\mathrm{T}^{\mathrm{j}} \mathcal{M} \subseteq \mathcal{M}$ whenever $\mathrm{T}^{\mathrm{j}} \mathrm{x} \in \mathrm{Orb}(\mathrm{T}, \mathrm{x}) \cap \mathcal{M}$.

Then T satisfies $\mathcal{M}$-hypercyclic criterion.

Proof. Let $(x, y) \in \mathrm{HC}(\mathrm{T} \oplus \mathrm{T}, \mathcal{M} \oplus \mathcal{M})$ such that $x$ satisfies the stated conditions. Since $x \in \mathrm{HC}(\mathrm{T}, \mathcal{M})$ (by Theorem 2.2), we show that the $\mathcal{M}$-hypercyclicity criterion is satisfied by the dense sets $D=D_{1}=D_{2}=$ $\operatorname{Orb}(T, x) \cap \mathcal{M}$. Since the operator $\mathrm{I} \oplus \mathrm{T}^{\mathrm{n}}$ commute with $\mathrm{T} \oplus \mathrm{T}$, then by Lemma 2.10, we have

$$
\left(x, T^{n} y\right) \in H C\left(T \oplus T, \mathcal{M} \oplus T^{n} \mathcal{M}\right)
$$

for all $n \in \mathbb{N}$. Since $y \in \operatorname{HC}(T, \mathcal{M})$ (by Theorem 2.2), then $\operatorname{Orb}(T, y) \cap \mathcal{M}$ intersects every open set in $\mathcal{M}$. Let $\mathrm{U}$ be an open neighborhood of 0 in $\mathcal{M}$, then there exist $u_{0} \in U \subseteq \mathcal{M}, r \in \mathbb{N}_{0}$ such that $u_{0}=T^{r} y$. It follows by (2.4) that

$$
\left(x, u_{0}\right) \in \mathrm{HC}\left(\mathrm{T} \oplus \mathrm{T}, \mathcal{M} \oplus \mathrm{T}^{\mathrm{r} \mathcal{M}}\right) .
$$

By condition $i$, we have $(0, x) \in \mathcal{M} \oplus \operatorname{Tr}^{r} \mathcal{M}$, then there exists $n_{0} \in \mathbb{N}$ such that

$$
\left(T^{n_{0}} x, T^{n_{0}} u_{0}\right) \in \operatorname{Orb}\left(T \oplus T,\left(x, u_{0}\right)\right) \cap\left(\mathcal{M} \oplus T^{r} \mathcal{M}\right)
$$

is arbitrarily close to $(0, x)$. Since $T^{n_{0}} \chi \in \mathcal{M}$, then $T^{n_{0}} \chi \in D$, and so $T^{n_{0}} \mathcal{M} \subseteq \mathcal{M}$ by condition $i$. By continuing the same process, we get a sequence $u_{k} \rightarrow 0$ in $\mathcal{M}$ and $n_{k} \in \mathbb{N}$ such that $T^{n_{k}} x \rightarrow 0, T^{n_{k}} u_{k} \rightarrow x$ and $T^{n_{k}} \mathcal{M} \subseteq \mathcal{M}$.

Let $C=\left\{i \in \mathbb{N}_{0}: T^{i} x \in D\right\}$, and let us define maps $S_{n_{k}}: D \rightarrow \mathcal{M}$ by $S_{n_{k}}\left(T^{i} x\right)=T^{i} u_{k} ; i \in C$ (by condition $i i$, it is clear that $S_{n_{k}}\left(T^{i} x\right) \in \mathcal{M}$ for all $\left.k \in \mathbb{N}_{0}, i \in C\right)$. Now, for all $T^{i} x \in D$, we have

$$
\begin{aligned}
& T^{n_{k}}\left(T^{i} x\right)=T^{i}\left(T^{n_{k}} x\right) \rightarrow 0, \\
& S_{n_{k}}\left(T^{i} x\right)=T^{i} u_{k} \rightarrow 0 \text { since } u_{k} \rightarrow 0, \\
& T^{n_{k}} S_{n_{k}}\left(T^{i} x\right)=T^{i} T^{n_{k}} u_{k} \rightarrow T^{i} x .
\end{aligned}
$$

for all $i \in C$. It follows that $T$ satisfies $\mathcal{M}$-hypercyclic criterion.

It follows by Theorem 2.11 above that hypercyclicity and subspace-hypercyclicity do not share the same properties. Therefore, one may wonder whether there are some more similarities and differences between hypercyclicity and subspace-hypercyclicity. 


\section{References}

[1] N. Bamerni, V. Kadets, A. Kılıçman, Hypercyclic operators are subspace hypercyclic, J. Math. Anal. Appl., 435 (2016), 1812-1815. https:/ / doi.org/10.1016/j.jmaa.2015.11.015 1

[2] N. Bamerni, A. Kilıçman, Subspace-hypercyclic weighted shifts, Oper. Matrices, 12 (2018), $205-214$. https:/ / doi.org/10.7153/oam-2018-12-13 1.5, 1.6

[3] F. Bayart, É. Matheron, Dynamics of Linear Operators, Cambridge University Press, (2009). 1

[4] J. P. Bés, A. Peris. Hereditarily hypercyclic operators, J. Funct. Anal., 167 (1999) 94-112. https:/ / doi.org/10.1006/jfan.1999.3437

[5] J. B. Conway. A Course in Functional Analysis, volume 96 of Graduate Texts in Mathematics, Springer-Verlag, (1990). 1,2

[6] M. De la Rosa, C. Read, A hypercyclic operator whose direct sum T $\oplus$ T is not hypercyclic, J. Operator. Theory. 61 (2009), 369-380. 1

[7] C. Kitai, Invariant Closed Sets for Linear Operators, Thesis, University of Toronto, (1982). 1

[8] C.M. Le, On subspace-hypercyclic operators, Proc. Amer. Math. Soc., 139 (2011), 2847-2852. 1

[9] B.F. Madore, R.A. Martínez-Avendaño, Subspace hypercyclicity, J. Math. Anal. Appl. 373 (2011), $502-511$. https://doi.org/10.1016/j.jmaa.2010.07.049

[10] H. Rezaei, Notes on subspace-hypercyclic operators, J. Math. Anal. Appl. 397 (2013), 428-433. https:/ /https:/ / doi.org/10.1016/j.jmaa.2012.08.002 1

[11] S. Rolewicz, On orbits of elements, Stud. Math. 32 (1969), 17-22. 1, 2

[12] T. Sorayya, Meysam A. On subspace-transitive operators, Int. J. Pure Appl. Math., 84 (2013), 643-649. 1

[13] T. Sorayya, Moosapoor. M, Subspace-Chaotic Operators and Subspace-Weakly Mixing Operators, Int. J. Pure Appl. Math., 78 (2012), 879-885. 1 